\title{
Conservative numerical boundary conditions
}

\author{
J. Burguete ${ }^{1}$, P. García-Navarro ${ }^{2}$ and J. Murillo ${ }^{2}$ \\ ${ }^{1}$ Suelo y Agua. Estación Experimental Aula Dei. CSIC. Zaragoza. Spain \\ ${ }^{2}$ Mecánica de Fluidos. CPS. Universidad de Zaragoza. Zaragoza. Spain
}

A recent technique for the numerical boundary conditions in 1D shallow water flow models is able to preserve the good properties of a conservative scheme used for the interior points. The implementation in a conservative scheme is shown in a test cases with exact solution and applied to the simulation of a real river flood wave leading to very satisfactory results.

\section{INTRODUCTION}

In order to reach the correct numerical solution in a general unsteady case, it is clear that the numerical scheme chosen for the interior points is as important as the method applied to discretize the boundary conditions. As for the boundary conditions, the starting point is the theory of characteristics. It provides a clear idea of the spacetime directions followed by the information, of the influence regions and of the required number of boundary conditons to have a well posed problem (Kreiss 1970). When, in the $x-t$ plane, a characteristic curve enters the computational domain, the region of influence is exterior to the domain and an additional condition is required. This is called a physical boundary condition. On the other hand, if the curve leaves the domain, the region of influence falls within the computational domain and the boundary condition depends exclusively in the interior variables. These are called numerical boundary conditions and their correct discrete representation is the main objective of this work.

The usual methods to discretize the numerical boundary conditions generate error in the global mass conservation. In this work, a recent method based on the integral form of the mass conservation equation extended to the full domain (global mass conservation) is also considered. It was first introduced in (Burguete et al. 2002; Burguete et al. 2006) and preliminarily used. The technique can be adapted to any conservative numerical scheme used for the interior points leading to a machine accuracy global mass conservation in all steady or unsteady situations.

\section{METHODOLOGY}

\subsection{Basic equations}

1D shallow water flows can be modelled by means of the Saint-Venant equations. The conservative form of such equations admits a vectorial expression as follows:

$$
\frac{\partial \mathbf{U}}{\partial t}+\frac{\partial \mathbf{F}}{\partial x}=\mathbf{H}
$$

with $\mathbf{U}$ the vector of conserved variables, $\mathbf{F}$ the fluxes and $\mathbf{H}$ the source terms:

$$
\mathbf{U}=\left(\begin{array}{c}
A \\
Q
\end{array}\right), \quad \mathbf{F}=\left(\begin{array}{c}
Q \\
\frac{Q^{2}}{A}+g I_{1}
\end{array}\right), \quad \mathbf{H}=\left(\begin{array}{c}
0 \\
g\left[I_{2}+A\left(S_{0}-S_{f}\right)\right]
\end{array}\right)
$$

where $A$ is the wetted cross section, $Q$ the discharge, $g$ the acceleration constant of gravity, $I_{1}$ and $I_{2}$ represent the pressure force integrals, $S_{0}$ the bed slope and $S_{f}$ the friction slope that is commonly modelled with the Gauckler-Manning law (Chow 1959). Defining the Jacobian of the conservative flux:

$$
\mathbf{J}=\frac{\partial \mathbf{F}}{\partial \mathbf{U}}=\left(\begin{array}{cc}
0 & 1 \\
c^{2}-u^{2} & 2 u
\end{array}\right)
$$


Here $u=\frac{Q}{A}$ is the flow velocity, $c=\sqrt{g \frac{A}{B}}$ the speed of the small surface waves and $B$ the wetted cross section top width. The eigenvectors of $\mathbf{J}$ are:

$$
\lambda_{1}=u+c, \quad \lambda_{2}=u-c
$$

and the matrices which diagonalize the jacobian:

$$
\mathbf{P}=\left(\begin{array}{cc}
1 & 1 \\
\lambda_{1} & \lambda_{2}
\end{array}\right), \quad \mathbf{L}=\left(\begin{array}{cc}
\lambda_{1} & 0 \\
0 & \lambda_{2}
\end{array}\right), \quad \mathbf{J}=\mathbf{P L P}^{-1}
$$

\subsection{Numerical scheme}

Having stated that our main goal is the derivation of a globally exact conservative scheme for the shallow water equations, conservative numerical methods are required both for the interior and the boundary points. The conservative form of the system of differential equations (1) can be solved by means of a conservative scheme. The following vector is defined for convenience (Burguete and García-Navarro 2001):

$$
\mathbf{G}_{i+(1 / 2)}=\left(\mathbf{H}-\frac{\delta \mathbf{F}}{\delta x}\right)_{i+(1 / 2)}
$$

The unified discretization of the friction term consists of discretizing flux, friction and other source terms in the equation in a similar form. A general conservative scheme with unified discretization can be expressed as (Burguete and García-Navarro 2001):

$$
\Delta \mathbf{U}_{i}^{p}=\Delta t\left(\mathbf{G}_{i-(1 / 2)}^{L}+\mathbf{G}_{i+(1 / 2)}^{R}\right)
$$

where $\mathbf{G}^{L, R}$ represents the wave discretization particular to each numerical scheme.

We shall definine the upwind matrices as:

$$
\mathbf{O}^{ \pm}=\frac{1}{2} \mathbf{P}\left(\begin{array}{cc}
1 \pm \operatorname{sign}\left(\lambda_{1}\right) & 0 \\
0 & 1 \pm \operatorname{sign}\left(\lambda_{2}\right)
\end{array}\right) \mathbf{P}^{-1}
$$

Then, the wave decomposition of the explicit upwind scheme is (Burguete and García-Navarro 2001):

$$
\mathbf{G}_{i+(1 / 2)}^{L}=\left(\mathbf{O}^{+} \mathbf{G}-\nu \frac{\delta \mathbf{U}}{\delta x}\right)_{i+(1 / 2)}, \quad \mathbf{G}_{i+(1 / 2)}^{R}=\left(\mathbf{O}^{-} \mathbf{G}+\nu \frac{\delta \mathbf{U}}{\delta x}\right)_{i+(1 / 2)}
$$

with $\nu$ the entropy artificial viscosity as in (Burguete et al. 2006).

\subsection{Numerical boundary conditions}

Numerical boundary conditions are those additional equations required to enable the correct numerical resolution at the boundary points. They carry some kind of information from the calculation domain. The method of global mass conservation (Burguete et al. 2002; Burguete et al. 2006) for 1D schemes is based on enforcing the integral form of the mass conservation extended to all the computational domain in combination with a conservative scheme for the interior points to generate the numerical boundary condition. This method is sensitive to the form the mass of the system is evaluated and to the physical boundary conditions.

In a domain discretized using $N$ cells, the mass increment $\Delta M^{n}$ in one time step is defined as:

$$
\Delta M^{n}=\sum_{i=1}^{N} \Delta A_{i}^{n} \delta x
$$

In a first step, a conservative scheme is used all over the domain neglecting contributions from outside cells. The cross section increments predicted in one time step are:

$$
\Delta A_{i}^{p}=-\frac{\Delta t}{\delta x}\left(\delta Q_{i+(1 / 2)}^{R}+\delta Q_{i-(1 / 2)}^{L}\right)
$$

The total numerical mass variation $\Delta M^{p}$ produced by the scheme considered is (Burguete, García-Navarro, and Aliod 2002):

$$
\Delta M^{p}=\sum_{i=1}^{N} \Delta A_{i}^{p} \delta x=\Delta t\left(Q_{1}^{n}-Q_{N}^{n}\right)
$$


Since the schemes used are conservative, this variation is only due to the boundaries and can be split into numerical contribution at the inlet $\Delta M_{i n}^{p}$ and at the outlet $\Delta M_{\text {out }}^{p}$ in the following form:

$$
\Delta M^{p}=\Delta M_{\text {in }}^{p}+\Delta M_{\text {out }}^{p}, \quad \Delta M_{\text {in }}^{p}=\Delta t Q_{1}^{n}, \quad \Delta M_{\text {out }}^{p}=-\Delta t Q_{N}^{n}
$$

If the physical boundary condition is, for instance, a certain mass input at the inlet $\Delta M_{i n}^{n}$ or at the outlet $\Delta M_{\text {out }}^{n}$, in order to ensure the global mass conservation of the scheme the numerical mass increment must be corrected. This is achieved by (Burguete et al. 2006):

$$
A_{1}^{n+1}=A_{1}^{p}+\frac{\Delta M_{i n}^{n}-\Delta t Q_{1}^{n}}{\delta x}, \quad A_{N}^{n+1}=A_{N}^{p}+\frac{\Delta M_{\text {out }}^{n}+\Delta t Q_{N}^{n}}{\delta x}
$$

Furthermore, for the the inner points:

$$
A_{i}^{n+1}=A_{i}^{p}, \quad Q_{i}^{n+1}=Q_{i}^{p}, \quad \forall i \neq 1, N
$$

\section{APPLICATIONS}

3.1 Test with analytical solution: discontinuous boundary conditions

One of the best forms to study the performance of the numerical schemes is to compare their numerical solution with the analytical solution in problems in which this exists. If a discontinuity is enforced to enter the upstream boundary of an ideal flat and smooth channel of uniform depth and discharge so that the flow properties on both sides obey the Rankine-Hugoniot equations, the discontinuity will propagate unchanged along the channel at a constant speed. From the initial conditions, the water depth $h_{i}$ and velocity $u_{i}$ are known in the channel. Imposing $h_{*}$ or $u_{*}$ at the boundary, the other variable and the front speed can be worked out solving these equations for the discontinuity. In the case of a prismatic rectangular channel and still water initial conditions $\left(u_{i}=0\right)$ (Burguete et al. 2006):

$$
U=\sqrt{g \frac{h_{*}}{h_{i}} \frac{h_{*}+h_{i}}{2}}, \quad u_{*}=\left(1-\frac{h_{i}}{h_{*}}\right) U, \quad Q_{*}=\sigma_{0}\left(h_{*}-h_{i}\right) U
$$

The propagation of a non-transcritical discontinuity in a rectangular channel $10 \mathrm{~m}$ width, with $h_{i}=0.4 \mathrm{~m}$, $h_{*}=1 \mathrm{~m}, Q_{*}=24.86021 \mathrm{~m}^{3} / \mathrm{s}$ and $U=4.143385 \mathrm{~m} / \mathrm{s}$ is used to study the accuracy of the resulting combinations of proposed numerical schemes for the interior points and methods for the numerical boundary conditions.

Figure 1 displays the numerical results obtained in this test using the explicit upwind scheme and the global mass conservation approach for the discretization of the upstream numerical boundary condition. This method achieves a reduction in the mass error to machine precision.

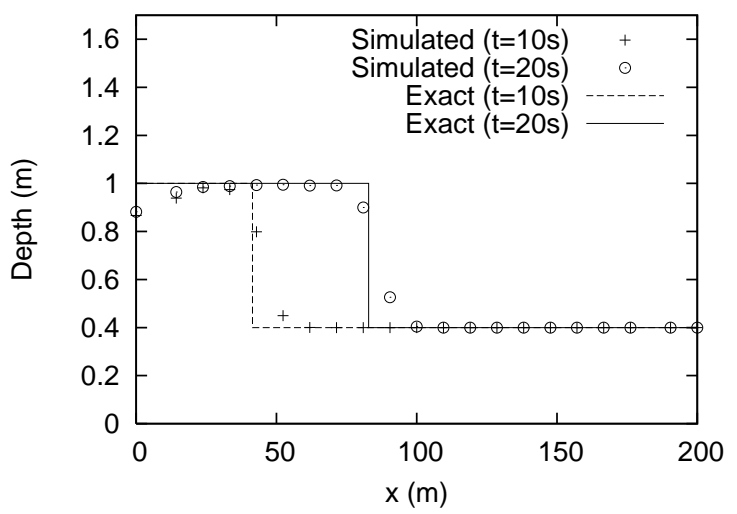

(a)

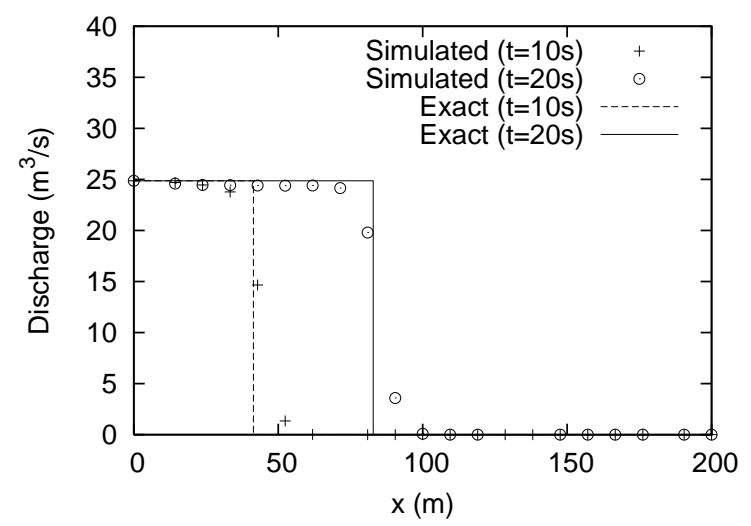

(b)

Figure 1: (a) Water depth, (b) discharge using the upwind scheme, $\delta x=10 m, C F L=0.9$ and the global mass conservation method for the upstream numerical boundary condition. 


\subsection{River flow application: Ésera River}

Ésera River is a Spanish river on the left bank of the Ebro River basin. It flows through a touristic mountain area of the Pirynees. A study has been carried on led by the interest to evaluate the risk of inundation in a few nearby camping sites. It is a typical, irregular and sloping mountain river (average slope around 4\%). 32 measured cross sections were used to define the $1 \mathrm{Km}$ long river reach bed form, a base discharge of $10 \mathrm{~m}^{3} / \mathrm{s}$ to state the initial flow conditions, and a high roughness Manning coefficient $n=0.03 \mathrm{~m}^{-1 / 3} s$ to model the stony bed according to Chow (1959).

Four flooding discharges of $236,344,414$ and $563 \mathrm{~m}^{3} / \mathrm{s}$, corresponding to return periods $10,50,100$ and 500 year respectively were assumed in the study. There was no field information either at the reach outlet so that, due the sloping character of the river bed, a critical outflow condition was assumed in case of downstream subcritical flow. At the upstream boundary, the global mass conservation method was used for the numerical condition discretization.

The calculation was performed in two steps. In a first step, the steady base flow was determined by sudden introduction of the base discharge over dry bed initial conditions. After convergence, this led to the initial conditions for the second run. Figure 2 is a plot of the water depth profiles corresponding to the base flow discharge and the four inundation discharges all flowing in steady state as computed with the explicit upwind scheme.

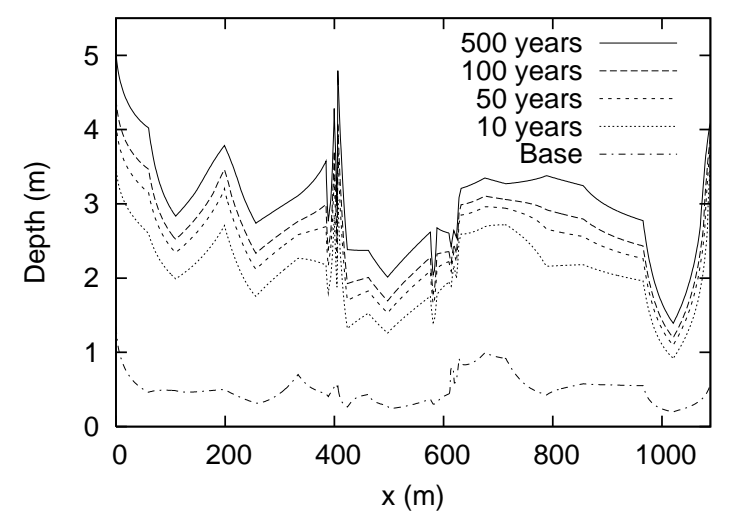

Figure 2: Several steady flow water depth profiles in the Ésera River reach as obtained from the upwind scheme using $\delta x=1 \mathrm{~m}$ and $\mathrm{CFL}=0.9$.

\section{CONCLUSIONS}

The global mass conservation method for the numerical boundary condition discretization is simple, is able to solve accurately unsteady flow with discontinuities at the boundary and leads to an exactly conservative solution when combined with any conservative explicit scheme for the interior points.

\section{References}

Burguete, J. and P. García-Navarro (2001). Efficient construction of high-resolution tvd conservative schemes for equations with source terms: application to shallow water flows. International Journal for Numerical Methods in Fluids 37(2), 209-248.

Burguete, J., P. García-Navarro, and R. Aliod (2002). Numerical simulation of runoff from extreme rainfall events in a mountain water cachtment. Natural Hazards in Earth System Sciences 2, 1-9.

Burguete, J., P. García-Navarro, and J. Murillo (2006). Numerical boundary conditions for globally mass conservative methods to solve the shallow-water equations and applied to river flow. International Journal for Numerical Methods in Fluids 51(6), 585-615.

Chow, V. T. (1959). Open channel hydraulics. New York: McGraw Hill.

Kreiss, H. O. (1970). Initial boundary value problem for hyperbolic systems. Communication of Pure and Applied Mathematics 23, 277-298. 